\title{
Georgios Bardanes, Metropolit von Kerkyra.
}

Der Zweck des vorliegenden Aufsatzes ist, einem Resultate V. Vasiljerskijs, das von dem durch Gelehrsamkeit und Kombinationsgabe ausgezeichneten Meister der russischen Byzantologie schon im J. 1885 sichergestellt, trotzdem aber, soweit ich sehe, bis auf den heutigen Tag den westeuropäischen Fachgelehrten vollständig unbekannt geblieben ist, endlich zu seinem Rechte $\mathrm{zu}$ verhelfen und damit einem landläufigen Irrtum ein Ende zu machen. Die Darlegung .Vasiljerskijs findet sich in seiner Abhandlung: „Die Erneuerung des bulgarischen Patriarchats unter dem Zaren Joann Asan II im J. 1235“, und zwar in der ersten Beilage, die überschrieben ist: „Über die Briefe des Metropoliten von Kerkyra Georgios, die in die kirchlichen Annalen des Baronius aufgenommen sind" (Journal des Minister. der Volksaufkl., Band 238, 1885, Aprilheft S. 224-233).

C. Baronius hat in seinen Annales ecclesiastici für die Jahre 1176-1188 elf Briefe eines Metropoliten von Kerkyra, namens Georgios, benutzt, die F. Mezio, Bischof von Termoli in Süditalien, nach einem ihm gehörigen griechischen Sammelkodex dem Herausgeber mitgeteilt hatte, leider nur in lateinischer Übersetzung. Zunächst wollen wir, ohne Berücksichtigung der üblichen rhetorischen Floskeln und Vergleiche, dem tatsächlichen Inhalte dieser elf Briefe etwas näher treten.

1) An den deutschen Kaiser Friedrich (1176 nach Baronius): Georgios dankt dem Kaiser, daB er ihn eines Briefes gewürdigt habe, den er übrigens wegen seiner Unkenntnis der lateinischen Sprache sich von dem gelehrten Untertanen des Kaisers, dem Richter und Magister Thomas, habe übersetzen lassen müssen, erklärt aber, die Forderung des Kaisers, ihm das Kastell von Kerkyra zu übergeben, als treuer Hirt der ihm anvertrauten Herde ablehnen zu müssen. Für den Kaiser könne ja bei dem gewaltigen Umfange seines Reichs der Besitz der kleinen Insel keine grobe Bedeutung haben. Deshalb appelliere er an das Rechtsgefühl des Kaisers, das diesen von einer Berautung seimes Nächsten abhaiten müsse, und bitte ihn, mit dem Gebieter des Georgios, dem erhabenen Komnenodukas Emmanuel, Er- 
barmen zu haben, da dieser sich eifrig um den Wohlstand seines Landes bemühe und auch stets mit größter Ehrerbietung des Kaisers gedenke.

2) An den Kaiser Emmanuel Dukas (1176): Georgios klagt, daB die kleine Besserung in seinem Befinden, die während der Anwesenheit des Kaisers eingetreten sei, zugleich mit dessen Weggang wieder aufgehört habe, und geht dann auf die schlimmen Nachrichten ein, die ihm der Kaiser brieflich mitgeteilt hat. Er verstehe sehr wohl das Gefühl der Trauer, das der für das Wohl seiner Untertanen besorgte Kaiser empfinde bei der Konspiration und Invasion der Barbaren und ihrem Prahlen, sie hätten obgesiegt und das Gewünschte erreicht. Aber der Sieg sei veränderlich und Gott werde den Kaiser an den bösen Nachbarn rächen. Der kaiserliche Befehl, die nötigen Schritte zur Sicherung des Kastells von Kerkyra zu tun, würde nach Kräften ausgeführt: Zisternen auf öffentliche Kosten errichtet, die waffenfähige Mannschaft mobilisiert, die Kriegsmaschinen instand gesetzt und der Hafen befestigt. Nachrichten aus Italien seien wegen des unterbrochenen Handelsverkehrs einstweilen nicht eingetroffen; doch hoffe er, in der ersten Fastenwoche dem Kaiser etwas Sicheres melden zu können.

3) An den Kaiser Komnenos (1176): Georgios drückt seine Freude über eine die Insel Kerkyra betreffende RegierungsmaBregel aus. Dadurch, daB der Kaiser die Verwaltung der Insel seiner Zwillingsschwester Komnena übergeben habe, liefere er wiederum einen Beweis seiner steten Fürsorge für das Wohl seiner Untertanen. Diese Maßregel ist getroffen ,in ipso imperii tui limine et cum primum paternae hereditatis factus es dominus".

4) An den Kaiser Friedrich (1178): Georgios rühmt die für die Widerstrebenden furchtbare, für die Willigen aber milde Herrschaft und Macht des Kaisers und motiviert sein kühnes Unterfangen, sich brieflich an den Kaiser zu wenden, damit, dab er von seinem Gebieter Emmanuel Komnenos Dukas beauftragt sei, dem Kaiser persönlich dessen Ehrerbietung zu bezeugen, worauf er sich weiter zum Papste begeben solle. Er sei nach einer stürmischen Seefahrt am 15. Oktober in Hydrus gelandet, dort aber erkrankt. Wenn er also dadurch und durch das Herannahen des Winters bis jetzt verhindert worden sei, den Auftrag seines Gebieters zu erfüllen, so werde er jedenfalls sofort beim Anbruch des Frühlings alles daran setzen, um sich dem Kaiser vorzustellen.

5) An Symeon, den Patriarchen von Theopolis (= Antiocheia, 1178): Georgios hat durch den Mönch Christophoros das 
Schreiben des Patriarchen zu der Zeit erhalten, wo er auf seiner Reise zum Papst in einer Stadt Italiens erkrankt war. Wie erfreulich die guten Nachrichten über das Befinden des Symeon seien, so betrübend sei anderseits die Kunde, daB die Ränke der Leute, die anderen Dogmen anhängen, dem Patriarchen Schwierigkeiten bereiteten und ihn hinderten, sein Licht an dem ihm bestimmten Orte leuchten zu lassen. Er hoffe aber zu Gott, daB dieser bald alles zum Besten wenden werde, "te videlicet, orbis lumen, in proprio constituens candelabro".

6) An den kaiserlichen Notar Johannes von Hydrus (1178): Georgios beklagt sich, daB, seitdem Johannes sich in die westlichen Länder begeben habe und beim Kaiser weile, er ihm keine Nachricht über sich gegeben und nichts über den Erfolg seiner Gesandtschaft geschrieben habe, auf der er vieler Leute Städte und Sitten (im Orient und im Okzident) gesehen habe. Mit Dankbarkeit gedenkt Georgios seines sechsmonatlichen Aufenthalts in Hydrus, wo er, am 17. November ins Haus des Johannes aufgenommen, dort die meiste Zeit krank gewesen sei und in Abwesenheit des auf der Gesandtschaftsreise befindlichen Johannes von dessen Hausgenossen die sorgfältigste Pflege erhalten habe. Seine Krankheit und die schlimme Jahreszeit hätten ihn so lange in Hydrus festgehalten. Als dann er und seine Gefährten endlich sich angeschickt hätten, die geplante Reise nach Rom zu unternehmen, habe ihm ein Schreiben seines Gebieters befohlen, sofort heimzukehren. So kehre er nun unverrichteter Sache zurück, wobei es ihn besonders betrübe, daB er die Hoffnung, den Kaiser zu sehen, aufgeben müsse.

7-8) An Nektarios, den Vorsteher des Klosters Casole (1179): Im ersten Briefe erklärt Georgios es für den gröBten Gewinn, $\mathrm{daB}$ er nach der Trennung vom hl. Vorsteher von Athen als Trost und Ersatz Nektarios gefunden habe und daB sie beide, wenn auch das weite Meer sie scheide, doch brieflichen Verkehr miteinander pflegen könnten. Er mache also mit diesem kurzen Brief den Anfang und hoffe sich in der Folgezeit ausführlicher mit ihm unterhalten zu können. Denn ihn verlange sehr nach der Rede und dem Verkehr mit Nektarios. Einen Vorgeschmack daron habe er ja einst, besonders in Byzanz, bekommen, als Pelagius jenen dahin mitbrachte und Georgios seinerseits als Abgesandter und Vertreter des Bischofs von Athen dort weilte. Im zweiten Briefe sagt Georgios, viele, die von dem früheren regen Briefwechsel zwischen ihnen beiden wüBten, wunderten sich, daB beide jetzt so lange schwiegen, und vermuteten sogar, das Freundschaftsiand sei gelöst. Gerade jetzt könne Nektarios doch viel von seiner Reise nach Rom erzählen, wo ẹ vor dem hl. Vater unerschrocken 
gegen seine Gegner aufgetreten sei, nicht wie einer, der einem Urteilsspruch übex sich entgegensehe, sondern wie ein Richter über die Irrlehren der Häretiker, so daB er beiden Parteien bewundernswert erschienen sei. Nektarios möge also seine Nektarlippen öffnen und erzählen. Auch hier erwähnt Georgios seine Krankheit und seine unerträglichen Schmerzen, die ihn an seiner Heilung verzweifeln lassen, und bittet zum SchluB, Herrn Johannes von ihm zu grüBen.

9) An den ökumenischen Patriarchen (1180): Georgios erklärt im Namen aller Prälaten des Westens, sie seien auf Befehl des Emmanuel Dukas bereitwilligst zusammengekommen und hätten das ihnen verlesene Schreiben des Patriarchen mit der größten Freude und Zustimmung angehört. ${ }^{1}$ )

10) An Nikolaos, den Richter von Hydrus (1180): Georgios gibt seiner Trauer über den Tod des Nektarios, des Abtes von Casole, Ausdruck. Die Tiefe seines Schmerzes werde Nikolaos, der Zögling des Verstorbenen, der von Kindheit an mit ihm zusammen gelebt habe, am besten ermessen können. Nektarios wird von Georgios mit begeisterten Worten nach seiner ganzen Persönlichkeit geschildert und gerühmt. Er sei eine Säule der Rechtgläubigkeit gewesen, ein Ruhm Italiens und ein Schmuck auch der berühmten Stadt Rom. Denn in dieser Stadt habe der trotz seines Alters noch rüstige Greis vor dem Papste zum Schutze seiner Bürgerschaft (periclitanti civitati auxiliatus) ausgezeichnet geredet und sei so den Seinigen als ein Bollwerk des Glaubens, den Gegnern aber wie ein verzehrendes Feuer erschienen. Wenn Georgios seine Abhandlungen, die in griechischer und lateinischer Sprache geschrieben seien, wieder lese, so komme es ihm vor, als ob er sich in der Gesellschaft des hl. Geistes befinde und über den Ausgang desselben (vom Vater allein) belehrt werde. Auch der hebräischen Sprache war Nektarios mächtig.

11) An Athanasios, den Patriarchen von Jerusalem (1188): Georgios habe oft den Wunsch gehegt, die hl. Stadt Jerusalem aufzusuchen, aber ans Krankenlager gefesselt, müsse er verzichten, es sei denn, daB die Gebete des Patriarchen seine Fesseln lösten. Von ganzem Herzen danke er dem Patriarchen, daB er ihn durch einen Brief geehrt und durch einige Abhandlungen getröstet habe. Er danke auch für das ihm übersandte Nardenöl.

Die Antwort des Patriarchen Athanasios auf diesen Brief bildet den AbschluB der hier kurz analysierten interessanten Korrespondenz.

1) Dieser Brief wird im nächsten Bande dieser Zeitschrift in einem Aufsatze "Christophoros von Ankyra als Exarch des Patr. Germanos II" genauer besprochen werden. 
Baronius hat die in den Briefen vorkommenden Namen Friedrich und Manuel auf den deutschen Kaiser Friedrich I Barbarossa (1152$1190)$ und den byzantinischen Kaiser Manuel Komnenos (1143-1180) bezogen. Aber ein Versuch Barbarossas, sich der Insel Korfu zu bemächtigen (vgl. 1), ist ganz undenkbar; dazu hätte er sich zuvor in Süditalien festsetzen müssen. Weder gleich bei seinem Regierungsantritt noch später hat der Kaiser Manuel die Insel Korfu der Verwaltung seiner Schwester übergeben ( $\nabla g l$. 3). Da Friedrich I noch nicht im Besitze des südlichen Italiens war und vom Herbst 1178 an nachweislich in Deutschland weilte, so lag für einen Bischof von Korfu der nächste Weg zu ihm keinesfalls zwischen Hydrus (Otranto) und Rom, wie es der 4. Brief darstellt. In den modernen Verzeichnissen der Patriarchen von Antiocheia begegnet uns allerdings unter dem J. 1178 ein Symeon, aber seine Existenz gründet sich ganz allein auf die von Baronius vorgetragene Datierung des 5. Briefes. ${ }^{1}$ ) Im J. 1178 konnte es in Otranto keinen kaiserlichen Notar geben (vgl. 6), da diese Stadt damals noch den normännisch-sizilischen Königen gehörte. Der im 7. Briefe erwähnte Pelagius ist der bekannte Legat des Papstes Innocenz III, der sich lange nach 1179, d. h. 1213/14 in Konstantinopel aufhielt. Den 11. Brief hat Baronius wohl nur aus dem Grunde auf das J. 1188 und keine frühere Zeit bezogen, weil er vor der Eroberung Jerusalems durch Saladin dort keinen griechischen Patriarchen neben dem lateinischen glaubte annehmen zu dürfen. Aber als solcher ist für die angegebene Zeit ein Athanasios durchaus nicht in anderen Quellen nachzuweisen.

Schon die angeführten Umstände beweisen die Unmöglichkeit der von Baronius durchgeführten Datierung der Briefe. Alles fügt sich aber aufs beste, wenn wir an den deutschen Kaiser Friedrich II (1212 -1250) und an Manuel den Despoten von Thessalonich und Epirus (1230-1240), der sich mit Vorliebe $\beta \alpha \sigma \iota \lambda \varepsilon v_{S}$ nannte und nennen lieB, denken. Bevor wir jedoch dies im einzelnen darlegen, müssen wir zusehen, wie sich die späteren Gelehrten zu der von Baronius aufgebrachten falschen Datierung gestellt haben.

L. Allatius, der an verschiedenen Stellen seiner Werke von dem Metropoliten Georgios von Kerkyra spricht, führt auch Bruchstücke aus zwei polemischen Schriften desselben gegen die Lateiner an, die er in einem alten Pergamentkodex der Barberina fand. Die erste ist

1) Aus einer syrischen Quelle erfahren wir, dab von 1164-1170 Antiocheia einen wirklich am Orte residierenden griechiscten Patriarchen besa B (vgl. W. Norden, Papsttum u. Byzanz S. 105). Am Ende dieses Jahrhunderts (etwa seit 1190) war der bekannte Kanonist Th. Balsamon wieder bloB Titularbischof dieser Stadt. 
laut ihrer Überschrift von Georgios in der Zeit abgefaBt, wo er im Kloster Casole (bei Otranto) krank lag, und war durch Fragen der

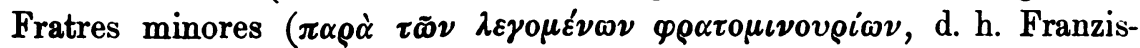
kaner) hinsichtlich des Fegfeuers hervorgerufen; die zweite bezog sich

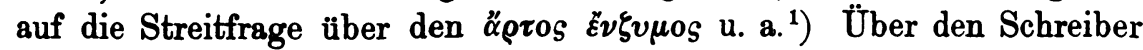

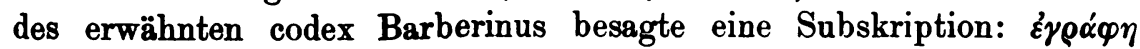

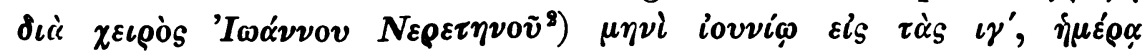

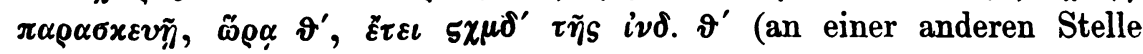
bietet Allatius $\left.\varsigma \varphi \mu \delta^{\prime}\right)$. Allatius identifizierte den Verfasser der polemischen Schrift mit dem Schreiber der oben erwähnten Briefe, womit er das Richtige traf; er beging aber den Fehler, daB er ohne Widerspruch auch die von Baronius gegebene Datierung (1176-1188) annahm, obwohl ihn davon schon die Erwägung hätte abhalten sollen, daB von den Minoriten doch frühestens seit 1209, wo die vom hl. Franziskus entworfene Regel zum erstenmal dem Papst Innocenz III vorgelegt wurde, die Rede sein konnte. Allatius hätte auch beachten sollen, $\mathrm{daB}$ zu dem von ihm angeführten Datum $5 \chi \mu \delta^{\prime}(=1136$, am 13. Juni) weder die 9. Indiktion noch der Wochentag (Freitag) paBt. Es ist, wie schon Fabricius bemerkte, $5 \psi \mu \delta^{\prime}(=1236)$ zu korrigieren, wobei alle einzelnen Angaben der Unterschrift vortrefflich miteinander stimmen.

Durch die eben erwähnten Bedenken veranlabt, gaben spätere Gelehrte (Oudin u. a.) die von Allatius angenommene Identität beider Metropoliten mit dem Namen Georgios auf und nahmen zwei verschiedene Personen an, von denen man den Briefschreiber mit Baronius ins Ende des 12., den durch Allatius bekannt gewordenen Polemiker aber in den Anfang des 13. Jahrhunderts verlegte. Und das ist bis auf den heutigen Tag die herrschende Ansicht geblieben (vgl. GBL 91 und 770).

Eine etwas abweichende, aber die Sache nur noch mehr verwirrende Stellung nahm in dieser Frage A. Mustoxidi ein, der ein dickes Buch über die Insel Korfu schrieb (Delle cose Corciresi. I. Corfu

1) Wie es sich jetzt herausgestellt hat, ist diese 2. Schrift oder vielmehr Aufzeichnung über den Verlauf einer in Konstantinopel abgehaltenen Dispu-

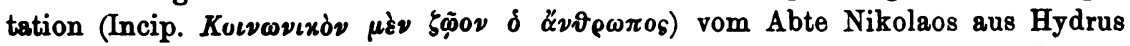
verfabt; sie wurde mit anderen Aufzeichnungen desselben Nikolaos im J. 1896 vom russischen Bischof Arsenij veroffentlicht (vgl. B. Z. VI 624).

2) Neretum, das heutige Nardo, lag Otranto gegenüber auf der apulischen Seite des Meerbusens. Die engen Beziehungen des Metropoliten Georgios zu den Nepertvoi (d. h. wohl Insassen des Klosters in Neretum) bezeugt auch ein Brief von ihm, den A. Mustoxidi (Delle cose Corciresi, Append. p. XLIX) nach dem cod. Paris. 1304 ediert hat. Der Brief gibt auf einige liturgische Fragen Antwort. 
1848) und dort außer anderen (griech.) Dokumenten, die den Metropoliten Georgios betreffen, auch die oben besprochenen latein. Briefe wiederabdruckte. Er trennte die drei ersten Briefe der von Baronius veröffentlichten Korrespondenz von den auf sie folgenden acht übrigen ab und kam so zu der Annahme von drei Bischöfen von Kerkyra mit dem gleichen Namen: Georgios I im J. 1148, wobei die von Manuel eingesetzte Regentin von Korfu (vgl. 3) nach Mustoxidi die Witwe des Kontostephanos ist; Georgios II Kupharas ${ }^{1}$ ) in den JJ. 1177-1181 und Georgios III Bardanes, der Polemiker, in den JJ. 1228-1236.

Unsere Kenntnis von Georgios Bardanes wurde dann durch die von Sp. Lampros besorgte Edition der Korrespondenz des Michael Akominatos (Athen 1879/80) bedeutend erweitert, da dort nicht wenige Briefe die Adresse des Bardanes tragen und ihn als Schüler und Freund des Athener Metropoliten erweisen. In der Dreiteilung der Metropoliten von Kerkyra mit dem Namen Georgios schlieBt sich Lampros (II S. 626) vollkommen der Ansicht von Mustoxidi an; nur den 7. Brief der Baroniusschen Sammlung, in dem vom Bischof von Athen die Rede ist, möchte Lampros abweichend von Mustoxidi dem Georgios II Kupharas wegnehmen und dem Georgios III Bardanes zuteilen. Den 8. Brief jedoch, der dieselbe Adresse trägt (Nektarios), läßt Lampros ruhig dem zweiten Georgios! Wie unkritjsch überhaupt ein solches Zerpflücken der äußerlich und innerlich sich als ein zusammenhängendes Ganze darstellenden Korrespondenz ist, braucht nicht erst bewiesen zu werden..$^{2}$ )

Nach diesen Irrgängen seiner Vorgänger hat nun V. Vasiljerskij der Frage die einzig richtige Wendung gegeben, indem er für den Zeitraum von der Mitte des 12. bis zur Mitte des 13. Jahrhunderts nur einen einzigen Metropoliten von Kerkyra mit dem Namen Georgios, den bekannten Georgios Bardanes, gelten lïßt und auf ihn auch die in den Annales ecclesiastici enthaltenen Briefe bezieht, deren Inhalt aufs beste zu seiner Person und seiner Zeit paßt.

1) Den Beinamen Kupharas bietet eine (wahrscheinlich auf Allatius zurückgehende) Notiz aus der noch unedierten „vierten Rede des Akindynos gegen Palamas". Diese nicht zu kontrollierende Notiz ist aber von Mustoxidi (p. 418) sehr unklar gefaBt. Es hat den Anschein, als ob es sich um einen Zeitgenossen des Palamas handelt, so daB also der erwähnte „Georgios Kupharas, später Metropolit von Kerkyra" vielmehr ins 14. Jahrhundert gehören würde.

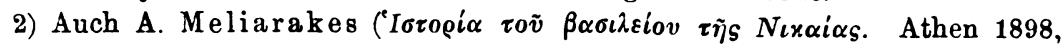
S. 204 Anm. 1) äuBert sich sehr anerkennend über die Hypothese Mustoxidis:

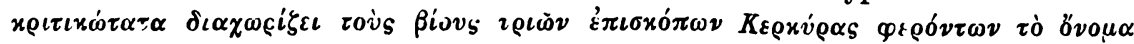

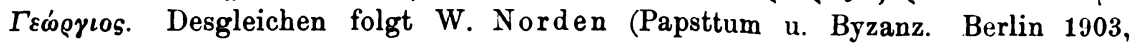
S. 112) ohne Bedenken der Ansicht Mustoxidis. 
Die Briefe fallen in die Zeit nach 1230, wo Manuel an Stelle seines von Asan II besiegten und gefangen genommenen Bruders Theodoros Dukas Angelos (1214-1230) Despot von Thessalonich wurde, und scheinen zeitlich nicht weit auseinander zu liegen (etwa 12301236). Auch ist wohl (im Gegensatz zu Vasiljevskij) daran festzuhalten, daB die Briefe, was ja das Zunächstliegende ist, in chronologischer Reihenfolge geordnet sind. Von dem Plane Friedrichs II, sich der Insel Korfu zu bemächtigen (vgl. 1) ist bei den Forschern, die seine Regierung in Spezialwerken geschildert baben (Winkelmann und Schirmacher) keine Rede, weil eben zu ihrer Zeit die Beziehung der Briefe bei Baronius zu der Epoche Friedrichs II noch nicht erkannt war. Die Zeit, in der dieser 1 . Brief geschrieben ist, läßt sich leider nicht mit Bestinmtheit feststellen. Die Stelle, die er in der Sammlung einnimmt, weist etwa auf das J. 1230 hin. Während der Wirren, welche die für den Despoten von Epirus so unglücklich verlaufene Schlacht bei Klokotnitza (April 1230) zunächst hervorrufen muBte, konnte Friedrich II am ehesten auf diesen Plan kommen. ${ }^{1}$ ) Vasiljerskij freilich meint, der Vorschlag Friedrichs, ihm die Insel zu übergeben, sei wahrscheinlich an den in Otranto weilenden Georgios gerichtet worden, in Beantwortung des 4. Briefes. Der magister et judex Thomas, der dem Georgios das Verständnis des kaiserlichen Schreibens vermittelte, war gewiß zugleich auch der mit der Überreichung desselben betraute Abgesandte des Kaisers. Es ist ohne Zweifel der magister justitiarius von Apulien Thomas de Aquino, comes Acerrarum, der in den Quellen zur Geschichte Friedrichs II in der Zeit von 1221-1235 mehrfach erwähnt wird. Den 2. Brief setzt Vasiljerskij ins J. 1237, als Manuel durch Michael II seiner Besitzungen im eigentlichen Epirus verlustig ging, sich in Thessalonich aber noch hielt. Aber dieser Zeitpunkt verstöBt nicht nur gegen die von uns festgehaltene chronologische Anordnung der Briefe, sondern stimmt auch nicht recht zu dem ganzen Tone und den einzelnen Ausdrücken des Briefes (molesta quaedam tibi contigisse significas). Speziell der Ausdruck, seine Gegner rühmten sich, quod praevaluerint adversus nos et superaverint portionem desiderabilem, scheint uns nicht das zu enthalten, was Vasiljerskij darin sieht, nämlich „den Verlust eines bedeutenden Teiles seiner Besitzungen“. Wir möchten deshalb unter den "benachbarten Barbaren“

1) Dazu kommt, daB eben im J. 1230 der 1223 aus Thessalonich vertriebene lateinische Konig dieser Stadt Demetrius von Montferrat anf seinem Sterbebette das Konigreich Friedrich II übertrug, was der Forderung Friedrichs II wegen Korfu eine gewisse reale Unterlage gab (vgl. Schirmacher, Gesch. Kaiser Friedrichs II Band III S. 93). 
lieber die Franken verstehen und einen ums J. 1231 erfochtenen Sieg derselben in einem uns weiter nicht bekannten Scharmützel annehmen. Die im 3. Brief erwähnte Schwester Manuels war die Gattin des Grafen Matteo, dessen Feudalbesitz die Inseln Kephallenia und Zante bildeten und den auch Manuels Vorgänger Theodoros als treuen Bundesgenossen erprobt und im Frühling 1229 als Gesandten an Friedrich II geschickt hatte. ${ }^{1}$ ) Der 4. Brief ist im Herbst 1231 geschrieben, zu welcher Zeit sich Friedrich II nachweislich in Italien aufhielt. Am 15. Oktober dieses Jahres kam Georgios in Otranto an und hielt sich dort, zumeist ans Krankenlager gefesselt, sechs Monate hindurch auf (bis zum April 1232). Während seiner Krankheit schrieb er, vom Franziskaner Bartholomaeus aufgefordert, seinen Traktat über das Fegfeuer (s. o. S. 608). Als er im Frühling 1232 endlich seine so lange unterbrochene Reise zum Kaiser und zum Papst wieder aufnehmen wollte, rief ihn ein Befehl des Despoten Manuel zurlick. Dieser überraschende Befehl hing offenbar mit dem uns aus anderen Quellen bekannten Wechsel in der von Manuel befolgten Politik zusammen. Während er nämlich kurz vorher zur Stärkung seiner Stellung den mächtigen Schutz des Papstes gesucht und sich bereit erklärt hatte, samt der Kirche seines Gebiets die päpstliche Oberhoheit anzuerkennen, wandte er sich jetzt an den orthodoxen Patriarchen von Nikaia mit der Bitte, der bisherigen Trennung der östlichen und westlichen Hälfte der griechischen Kirche ein Ende zu machen und auch ein politisches Bündnis zwischen ihm und dem Kaiser Joh. Batatzes zu befürworten (vgl. Meliarakes, 'Iбचopia S. 291). Der Patriarch von Antiocheia Symeon (vgl. 5) wird ebenso wie der Patriarch von Jerusalem Athanasios (vgl. 11) in dem Berichte über die Erneuerung des bulgarischen Patriarchats (1235), zu der beide schriftlich ihre Zustimmung gaben, erwähnt. Symeon wird außerdem in einem arabischen Verzeichnis der Patriarchen von Antiocheia an dritter Stelle hinter Balsamon aufgeführt (Joakeim, Dorotheos, Symeon), und Athanasios kommt wiederholt in der Lebensbeschreibung des hl. Sabbas von Serbien vor, der zwei Pilgerreisen ins Heilige Land unternahm $(\dagger$ 1235). Auch den Notar Johannes von Otranto (vgl. 6) können wir aus anderen Quellen dieser Zeit nachweisen. Es ist der seit 1227 in offiziellen Dokumenten vorkommende Johannes imperialis aulae notarius, der im J. 1240 als magister justitiarius curiae Bittschriften auf den Namen des Kaisers Friedrich entgegennimmt. Er ist auch mit

1) Über die Schicksale des Grafen Matteo von Zante vgl. Hopf, Gesch. Griechenlands S. 257/8. Im cud. Petiopol. 200 foi. $66^{\circ}$ and im cod. Hierosolym. 276 fol. 137v ist der diesem Matteo im J. 1228 geleistete Vasalleneid Benedikts, des latein. Bischofs von Kephallenia, in griech. Sprache erhalten. 
dem von K. Krumbacher (GBL 769) nachgewiesenen Verfasser des Gedichts auf die Unterwerfung der Stadt Parma identisch. Aus dem 7. Briefe (an Nektarios) erfahren wir, daB im J. 1213/4, als der römische Legat Pelagius mit Nektarios sich in Konstantinopel befand, auch Georgios Bardanes als Abgesandter des Michael Akominatos sich dort aufhielt. Eine Bestätigung dieser Nachricht und zugleich die Mitteilung, daB Bardanes im nächsten Jahre wiederum eine Seefahrt (nach Nikaia?) unternommen hat, enthält ein Brief des Mich. Akominatos

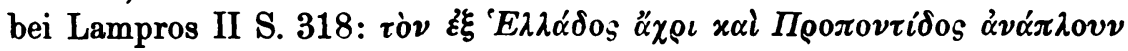

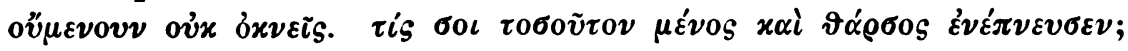

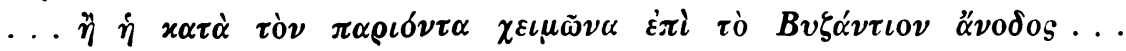
Einen dritten Brief des Bardanes an Nektarios (in griech. Sprache) hat Mustoxidi nach dem cod. Laurent. IX 16 veröffentlicht (Append. p. XLIII); in ihm bittet Georgios um eine Abschrift der,$\delta\llcorner\alpha \lambda o \gamma \iota x \tilde{\omega} \nu$

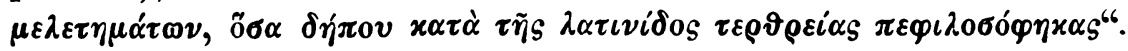
Von diesen und den im 10. Briefe erwähnten polemischen Traktaten des Nektarios scheint nichts auf uns gekommen zu sein; über seine dichterische Tätigkeit vgl. K. Krumbacher (GBL 770, 2). Auch Nikolaos, Richter in Hydrus, der Adressat des 10. Briefes und Schüler des Nektarios, läBt sich aus anderen Dokumenten nachweisen. Es ist Nicolaus de Girachio (in Kalabrien), der 1231 noch als einfacher Finanzbeamter (rationalis) in Apulien, 1239 aber schon als justitiarius aulae Udronti vorkommt. Natürlich ist er von Nikolaos von Hydrus, dem Vorgänger des Nektarios in der Abtswürde von Casole und Begleiter des Kardinals Benedikt (1206), dessen polemische Aufzeichnungen Arsenij ediert hat (vgl. oben S. 608 Anm. 1), zu unterscheiden, wohl aber identisch mit dem Nikolaos, von dem der cod. Laurent. V 10 verschiedene Epigramme enthält (GBL 769). Sein in der Überschrift dieser Gedichte genannter Vater, der Maïstros Johannes $\delta \varepsilon \pi i \tau \tilde{\nu} \nu \delta \varepsilon \dot{\gamma} \sigma \varepsilon \omega \nu$, ist der oben besprochene Adressat des 6 . Briefes.

$\mathrm{DaB}$ übrigens auch Georgios Bardanes sich als Dichter betätigt hat, bezeugen sieben jambische Gedichte ron ihm im leider stark verstümmelten cod. Cryptensis Z $\propto$ XXIX; vgl. Krumbacher, GBL 770, 4 und L. Sternbach, Observationes in Georg. Corcyraeum. 1899 (Eos V 113-119).

Aus dem oben Dargelegten ergibt sich, daB die von Vasiljevskij an die richtige Stelle gesetzten Briefe bei Baronius einen reichhaltigen Stoff zur Lebensbeschreibung und Charakteristik des Georgios Bardanes bieten, und zwar besonders für die letzte Zeit seines Lebens, wo er als Metropolit der Insel Korfu unter der orthodoxen Geistlichkeit des Westens eine hervorragende Stellung einnahm und wiederholt als ihr 
Bevollmächtigter sich brieflich an den Patriarchen von Nikaia wandte, und ebenso von dem Despoten Manuel $^{1}$ ) eines besonderen Vertrauens gewürdigt und mit wichtigen diplomatischen Aufträgen betraut wurde, und auch mit den aus Unteritalien stammenden und als höhere Beamte des Kaisers Friedrich II eine bemerkenswerte Rolle spielenden Griechen und mit den Patriarchen von Antiocheia und Jerusalem rege Beziehungen unterhielt. So ergänzen also diese Briefe in sehr erwünschter Weise das, was wir bisher aus der Korrespondenz des Michael Akominatos

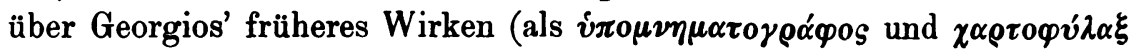
von Athen) und aus den von Vasiljevskij in den Epirotica (Viz. Vrem. 3, 1896, 248-263) edierten Dokumenten über seine darauf folgende Tätigkeit in Grebena, seine Aussichten auf den Bischofsstuhl von Bonditza und schlieBlich seine Wahl zum Metropoliten von Korfu gegen Ende des J. 1219 wußten. ${ }^{2}$ ) $\mathrm{Da}$ dies aber von Lampros in seiner Ausgabe des Mich. Akominatos (II S. 624-626) und von Meliarakes

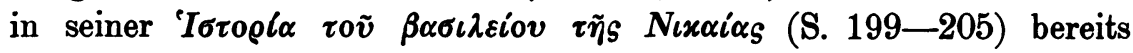
zusammengestellt und genügend beleuchtet ist, brauchen wir hier zum SchluB nur noch einen Punkt zu berühren, nämlich die Herkunft des Georgios Bardanes. Meliarakes meint, daB Bardanes ein Grieche aus Ägypten gewesen sei, aber diese Vermutung beruht nur auf einem Mißverständnis; denn der von Mich. Akominatos in einem Briefe an Bardanes gebrauchte Ausdruck: ,

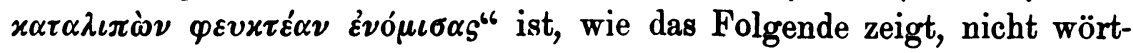
lich, sondern allegorisch aufzufassen. Vielmehr weist der Beiname

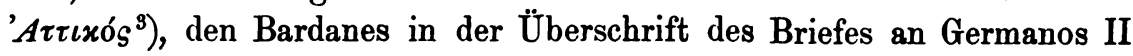
im cod. Barocc. 131 erhält (ed. A. Mustoxidi, Append. p. LV), darauf hin, daB er aus Athen stammte, wo auch seine Mutter und seine Brüder lebten; der von Meliarakes ausgesprochene Gedanke, er hahe

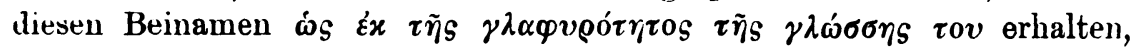
scheint sich weniger zu empfehlen.

Riga.

Ed. Kartz.

1) Sofort bei seirem Regierungsantritt (1230) bestätigte Manuel in einer Bulle (ed. Mustoxydes im Hellenomnemon S. 208) die alten Privilegien der Metropole und der Bevölkerung Korfus, was zwei Jahre früher auch Theodoros Dukas Angelos getan batte (abgedruckt bei Mustoxidi, Delle cose Corcir. Append. p. LVI).

2) Meliarakes setzt die Wahl nicht richtig ins J. 1220, indem er auBer acht läBt, daB es sich um den Dezember der 8. Indiktion handelt.

3) Die Angabe im Titel des ersten Gedichts des Georgios im Cryptensis

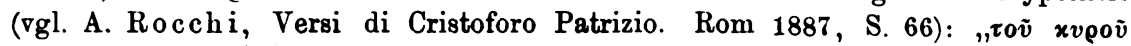

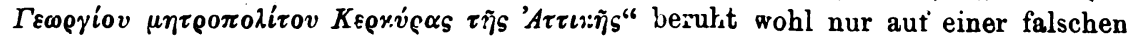

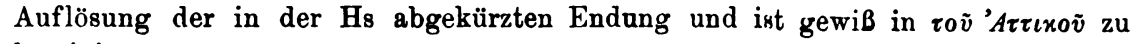
korrigieren.

Byzant. Zeitschrift XV 3 u. 4. 